\title{
Metascreen-Based Acoustic Passive Phased Array
}

\author{
Yong Li $1,{ }^{1,2, *}$ Xue Jiang, ${ }^{3}$ Bin Liang, ${ }^{3, \dagger}$ Jian-chun Cheng, ${ }^{3, *}$ and Likun Zhang ${ }^{4, \S}$ \\ ${ }^{1}$ CNRS, Institut Jean Lamour, Vandouvre-lès-Nancy F-54500, France \\ ${ }^{2}$ Université de Lorraine, Institut Jean Lamour, Boulevard des Aiguillettes, BP: 70239, \\ Vandoeuvre-lès-Nancy 54506, France \\ ${ }^{3}$ Key Laboratory of Modern Acoustics, MOE, Department of Physics, \\ Collaborative Innovation Center of Advanced Microstructures, \\ Nanjing University, Nanjing 210093, People's Republic of China \\ ${ }^{4}$ Department of Physics and Center for Nonlinear Dynamics, University of Texas at Austin, \\ Austin, Texas 78712, USA
}

(Received 11 January 2015; revised manuscript received 24 May 2015; published 6 August 2015)

\begin{abstract}
Conventional phased arrays require a large number of sources in forming a complex wave front, resulting in complexity and a high cost to operate the individual sources. We present a passive phased array using an acoustic metascreen that transmits sound energy from a single source and steers the transmitted wave front to form the desired fields. The metascreen is composed of elements that have a discrete resolution along the screen at an order smaller than the wavelength, allowing for fine wave-front shaping beyond the paraxial approximation. The performance is verified in experiment by forming a self-bending beam. Our metascreen-based passive array with its simplicity and capability has applications in places where conventional active arrays are complex and have limitations.
\end{abstract}

DOI: 10.1103/PhysRevApplied.4.024003

\section{INTRODUCTION}

Various areas call for the desire to form wave fields of a specific property such as nondiffracting [1], self-bending [2], twisted [3], or focused wave front. All of these wave fields are constructed from solutions of wave equations in either the paraxial or nonparaxial region. According to the Huygens principle, all wave fields can be assembled from the wave-front shape of elementary waves. This is the principle of a phased source array composed of an array of elementary sources with proper relative phases to bend, steer, or focus the energy of a wave front that forms the desired wave fields. The conventional phased array, however, requires a large aperture and, hence, a large number of sources in forming a complex wave front or nonparaxial wave beams, leading to high cost and complexity in the electronics required to operate the individual sources of the array.

One can avoid the complexity of the conventional phased array by manipulating the wave front of a single source using a passive structure that allows the penetration of the wave energy and provides a local phase delay to steer the transmitted wave front [cf. Fig. 1]. The elementary unit of the structure then needs to have the capability of (i) shaping the phase over a full $2 \pi$ range, (ii) overcoming impedance mismatching at the unit for the wave energy to penetrate through the structure with a high transmission, and

\footnotetext{
*yong.li@univ-lorraine.fr

†liangbin@nju.edu.cn

\#jccheng@nju.edu.cn

\$zhang@chaos.utexas.edu
}

(iii) holding a fine discrete resolution along the array regarding the spatial aliasing effect, especially in forming nonparaxial fields [4]. In optics, these functions can be feasibly fulfilled by beam-shaping devices or structures like spatial light modulators [5] or recently emerged metasurfaces [6-8]. The manipulation is beneficial from the polarization and the plasmonic resonance in optical waves, which do not yet have mechanical counterparts in acoustic waves.

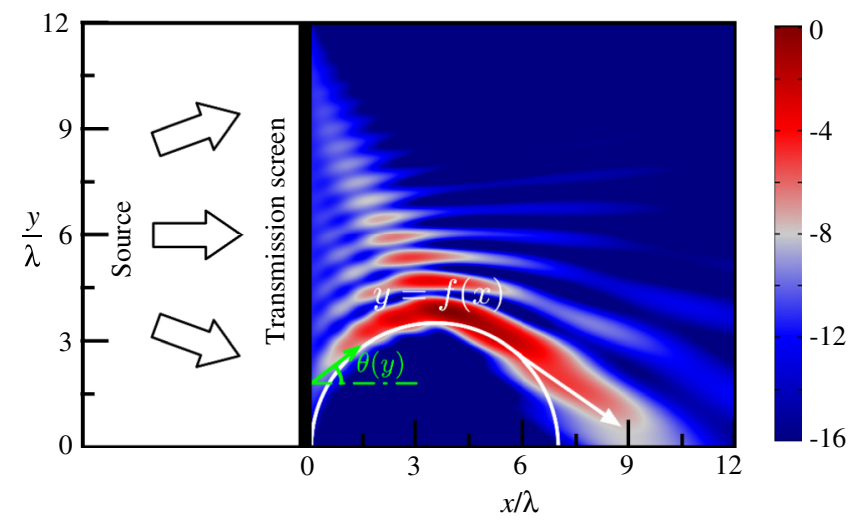

FIG. 1. Illustration of penetrating sound energy from a primary source through a passive screen at $x=0$ (the thin black box) that adds a phase shift $\phi(y)$ on transmitted fields to shape wave-front propagation along a desired path $y=f(x)$. The illustrated sound field (pressure level in units of decibels and referenced to the maximum value) is a self-bending beam whose path is the white arc line of radius $r=3.5 \lambda$ ( $\lambda$ is the sound wavelength) shaped from $\phi(y)=-k(y-2 k r \sqrt{y / r})$. 
The manipulation in acoustics is limited by a trade-off between the impedance mismatching determining the transmission efficiency and the refractive index playing a dominant role in phase shaping [9]. It is a further challenge to overcome this trade-off and, meanwhile, hold a fine spatial resolution. As such, the existing acoustic metamaterials [10-15] and metasurfaces [16-22], despite their capability of providing parameters unavailable in nature, have limited performance on the achievement of all three aforementioned functions.

To this end, we present a design and realization of an acoustic passive metascreen that has the extreme capabilities of high transmission, full phase control, and deep subwavelength spatial resolution that shape a transmitted wave front into wave fields of high quality in both the paraxial and nonparaxial regions.

\section{ILLUSTRATION}

Imagine that a sound field radiated by a primary source transmits through a planar screen [cf. Fig. 1]. The screen provides a local phase shift $\phi(y)$ on the incident acoustic field, consequently forming the transmitted sound to propagate along a desired path $y=f(x)$. Following ray tracing, the relationship between the phase shift profile $\phi(y)$ and the sound path $y=f(x)$ is $d \phi(y) / d y=-k \sin \theta(y)$, where $k$ is the sound wave number in the medium, and $\theta(y)$ is the angle of the path with the path slope $\tan \theta=f^{\prime}(x)$ [cf. Fig. 1]. As an example, the sound field illustrated in Fig. 1(a) is a beam that has a half-circle path $f(x)=\sqrt{r^{2}-(x-r)^{2}}$ centered at $(x, y)=(r, 0)$, and the desired phase-shift profile for generating such a bending beam from a normally incident plane wave front is $\phi(y)=-k(y-2 k r \sqrt{y / r})$. This phase profile illustrates the requirement for the full control of a transmitted wave front on the screen. The requirement, in addition to the efficient transmission, is the ability to yield a phase shift that rapidly varies along the screen in the $y$ direction. The variation requires the screen to have a fine spatial resolution when using discrete structures along the $y$ direction to minimize the spatial aliasing effect.

\section{DESIGN}

We use elements of a hybrid structure shown in Fig. 2(a) to construct the transmission screen. The structure consists of deep subwavelength cavities, which are acoustic HRs, and a straight pipe at the open side of the HRs. The HRs act as lumped elements providing an effective acoustic reactance to shift the phase of the incident acoustic field [9], but the phase shift provided by a single HR is limited within a small range.

Hereby, a series connection of four HRs is employed to achieve a wide range of phase shifts [cf. Fig. 2(a)]. We use (a)
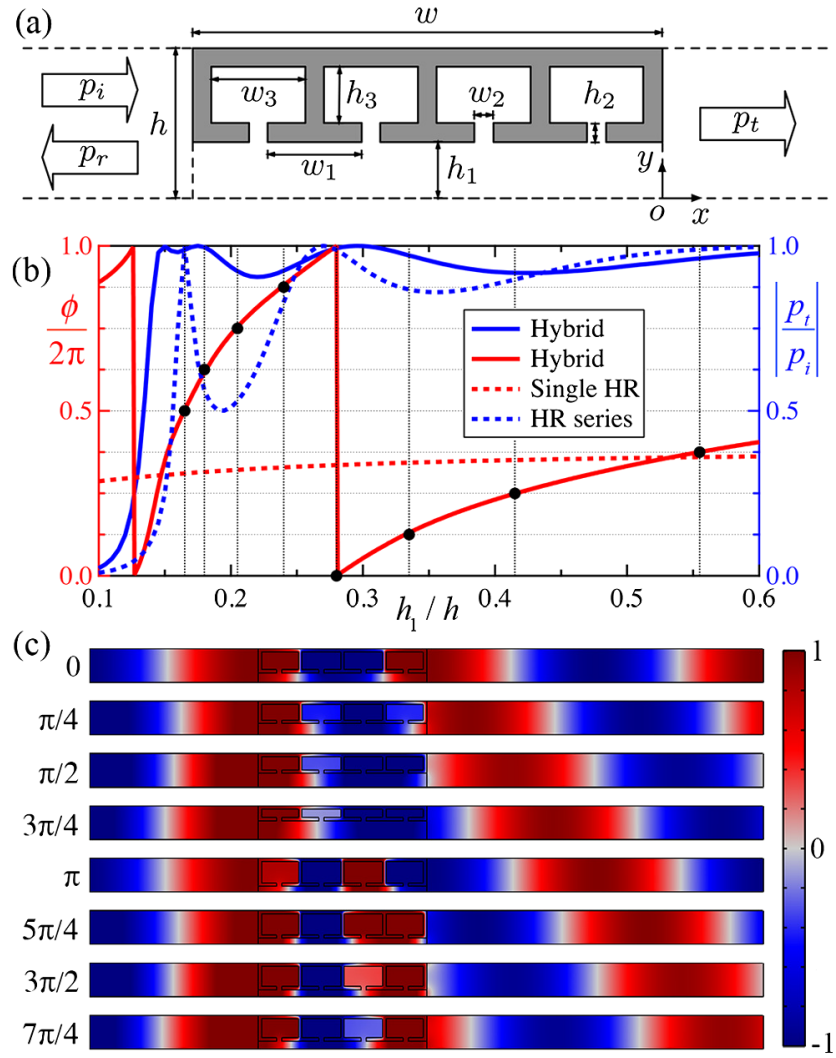

FIG. 2. The passive screen is stacked up by an array of passive elements. (a) Each element is designed to have a hybrid structure consisting of a straight pipe of height $h_{1}$ and four Helmholtz resonators (HRs) of height $h_{3}$; the element dimensions are $w=\lambda / 2$ and $h=\lambda / 10$. (b) Phase shift (red, solid) and transmission rate (blue, solid) of the hybrid structure as a function of height ratio $h_{1} / h$ (or, correspondingly, $h_{3} / h$ ), and a comparison with that of Helmholtz resonators (red and blue dashed). Other parameters are fixed as $\left(w_{1}, w_{2}, w_{3}\right)=$ $(0.22,0.03,0.225) w$ and $h_{2}=0.1 h$ that are not too small to minimize the viscous effect. (c) Simulated sound transmission through individual elements for an equally increased phase shift with a step of $\pi / 4$, corresponding to the eight dots in (b) with $h_{1} / h=0.28,0.33,0.41,0.55,0.16,0.18,0.20$, and 0.24 . Plotted snapshot of the sound field is referenced to the amplitude of incident plane waves.

HRs in a series with a tunable height $h_{3}$ to span the phase shift over a full $2 \pi$ range. However, there is an impedance mismatch between the Helmholtz resonators and the surrounding medium that leads to a low transmission of the sound [9]. The series connection of HRs inevitably enlarges the impedance mismatch as compared with a single HR with the sounding medium. As a result, this series connection leads to an even lower transmission.

In order to obtain a high transmission, we propose a hybrid structure by coupling the series of HRs with a straight pipe of a tunable height $h_{1}$ and a fixed width $w=$ $\lambda / 2$ [cf. Fig. 2(a)]. With the $\lambda / 2$ length, this straight pipe will effectively match the acoustic impedance, which is 
based on the Fabry-Perot resonance [23,24] and, hence, effectively enhances the sound transmission.

The way that we design a structure in such a hybrid manner allows us to keep a deep subwavelength resolution along the screen direction, i.e., $h \ll \lambda$ [cf. Fig. 2(a)]. Here we choose the dimension $h$ to be an order smaller than the sound wavelength, $h=\lambda / 10$, such that there are ten elements over each wavelength range along the screen. That is a reasonable resolution to achieve a fine manipulation of the wave front beyond the paraxial approximation.

We fix the unit dimension $h$ for the convenience of stacking the units together. Therefore, by tuning the cavity height $h_{3}$, the pipe height $h_{1}$ changes as well (although one can alternatively fix $h_{1}$ but change the unit dimension $h$ with the cavity height $h_{3}$ ). We use as many as four HRs in a series, which is the minimum number needed to provide enough resonances with the pipe to maintain the transmission performance, as we verify through our simulations.

\section{SIMULATIONS}

\section{A. Dependence on structural parameters}

We simulate the phase shift and transmission coefficient of the proposed structure as a function of the tunable pipe (or cavity) height ratio, $h_{1} / h$ (or $h_{3} / h$ ) in Fig. 2(b). The simulation is for a fixed element height $h=\lambda / 10$ and normally incident plane waves [25]. The transmission (blue solid line) is up to 0.9 once $h_{1} / h>0.143$, where the phase shift (red solid line) spans the whole $2 \pi$ range when tuning $0.15 \leq h_{1} / h \leq 0.6$. For comparison, also plotted is the phase shift (red dashed line) yielded by a single HR, illustrating its small range of phase shift. A comparison of the transmission with that of the HRs series (blue dashed curve) shows that the coupling of HRs with the pipe for the Fabry-Perot resonance greatly enhances the transmission over a wide range of $h_{1} / h$.

We use eight values of $h_{1} / h$ shown by the eight dots in Fig. 2(b) to achieve eight steps of an equally spaced phase shift for practical realization. The simulations of the transmission through these eight individual elements are illustrated in Fig. 2(c). The simulated primary field is a plane wave normally incident from the negative $x$ side. The computed fields show the desired phase shift equally spaced from 0 to $2 \pi$ and also a high transmission that is larger than 0.92 .

\section{B. Transmission spectra}

The above simulations are for the Fabry-Perot resonances, where the frequency equals the resonant frequency (denoted by $f_{0}$ ) associated with $w=\lambda / 2$. Here we further simulate the transmission spectra of the eight elementary units. The transmission coefficient shown in Fig. 3(a) is
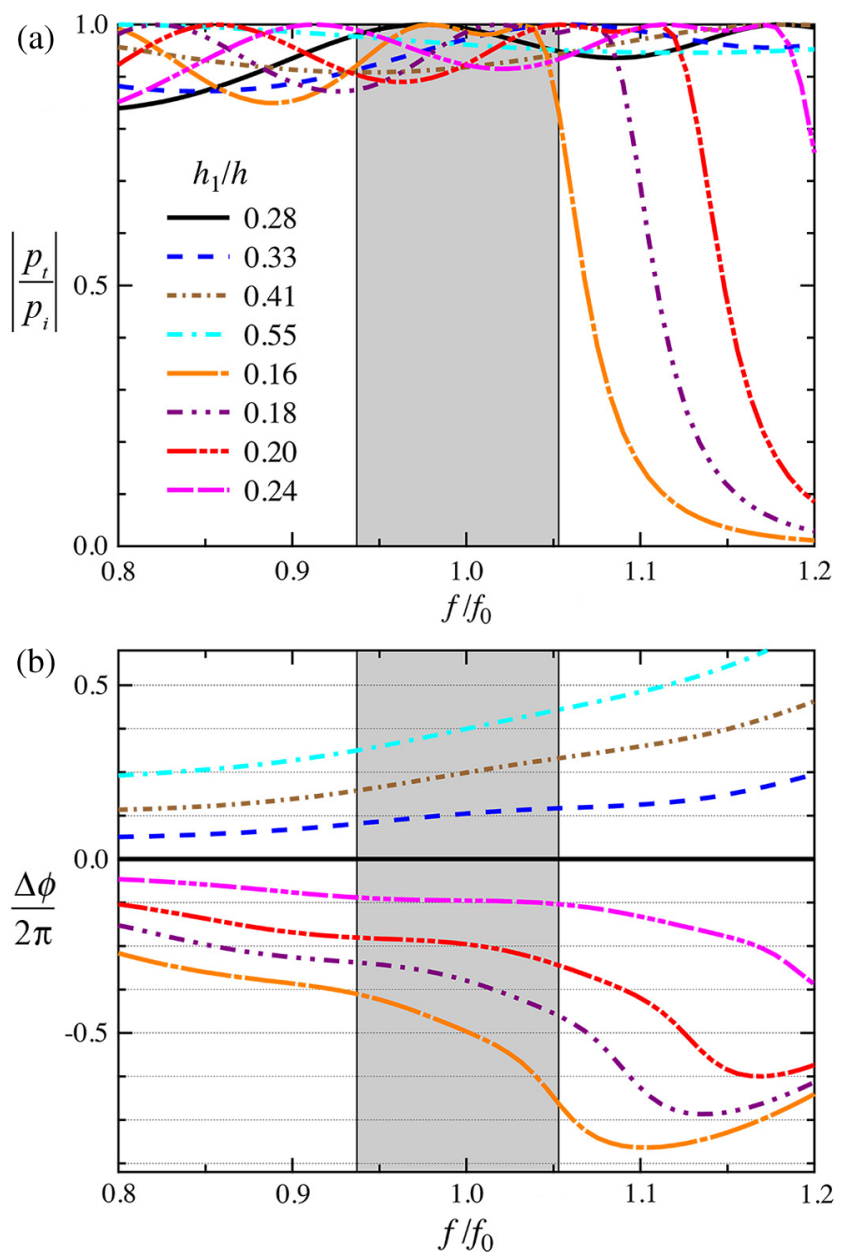

FIG. 3. (a) Transmission coefficient and (b) phase shift as a function of frequency $f$ for the eight elements presented in Fig. 2(c), showing that the elements work effectively within a finite band of frequency $f$ around the Fabry-Perot resonant frequency $f_{0}$ (corresponding to $w=\lambda / 2$ ) shown by the gray area for $f / f_{0} \in[0.937,1.053]$ (see text for details). The phase shift in (b) is shown as the difference of the phase shift from that provided by the element with $h_{1} / h=0.28$.

considerably high over a relatively broad frequency range. This is a result of Fano-like resonances supported by the coupling of the straight pipe with the HRs in the hybrid structure of the units [cf. Fig. 2(a)]. Each unit containing four HRs gives four coupling resonances and, hence, leads to four transmission peaks at the transmission spectra over a relatively broad frequency range [this range is not completely covered in Fig. 3(a)]. Even so, the transmission coefficient drops to $80 \%$ when $f / f_{0}$ increases to 1.053 . On the other hand, the spanning range of the phase shift of these eight elements narrows to $80 \%$ of $2 \pi$ when $f / f_{0}$ decreases to 0.937, as shown in Fig. 3(b). As a result, the performance of the elements is limited in a finite band of frequency range between these two cutoffs. Within this band, $0.937 \leq f / f_{0} \leq 1.053$, the units should have a reasonably good performance. 


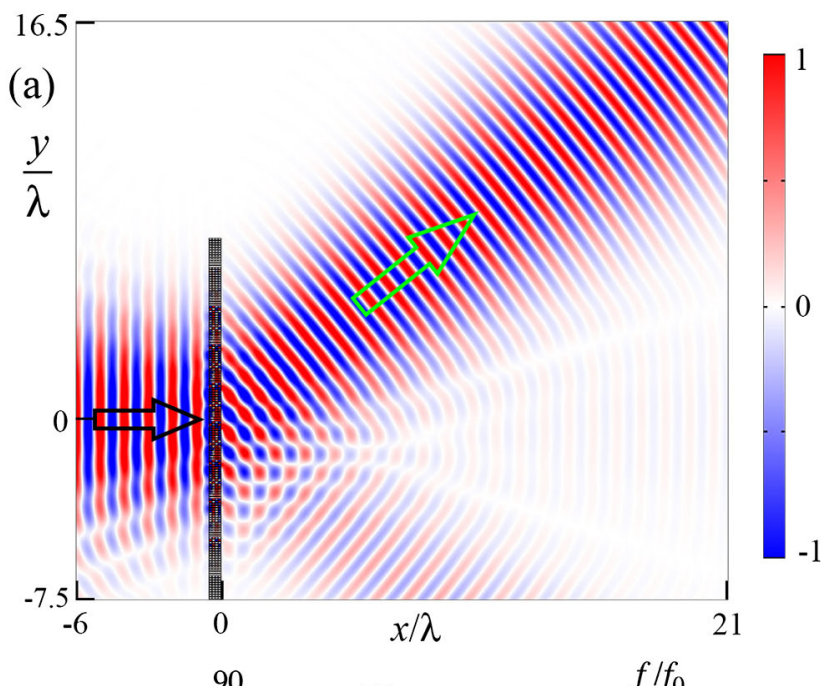

(b)

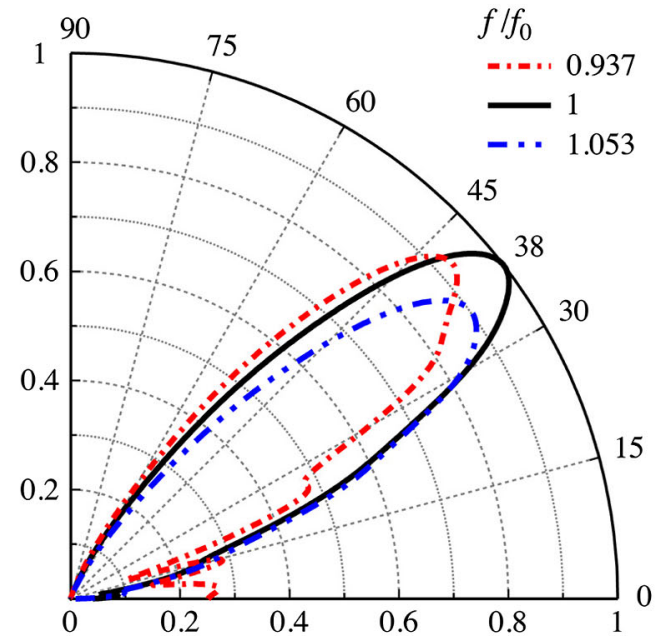

FIG. 4. Redirection of acoustic beams by a 150-element screen that provides a stair-step phase profile along the screen. (a) Sound fields simulated for the screen that redirects a normally incident acoustic beam of $f=f_{0}$ at an angle of $38^{\circ}$ (denoted by green arrow) away from its incident direction (denoted by black arrow). (b) Polar directivity graph of the redirected beams measured at ten wavelengths away from the center of the screen, at $f=f_{0}$ (solid curve), and at the two cutoff frequencies in Fig. 3 (dot-dashed curves).

\section{Redirection of acoustic beams}

To examine the performance, we numerically apply our designed units to redirect the propagation of an acoustic beam. Here, a 150-unit screen used for the redirection provides a stair-step discrete phase profile that resembles the desired linearly gradient phase profile, $\phi(y)=$ $-k \sin (\theta) y$, where $\theta$ is the designed redirection angle $\left(38^{\circ}\right.$ herein), and $k$ is the wave number associated with the FabryPerot resonance at $f_{0}$. The simulated results reveal that the redirected beam is in a good shape as the incident beam [cf. Fig. 4(a)] and propagates along the designed angle of $38^{\circ}$ when $f=f_{0}$ [cf. solid curve in Fig. 4(b)], but at cutoff frequencies, both the beam amplitude and redirected angle are deviated a bit [cf. dot-dashed curves in Fig. 4(b)].

\section{EXPERIMENT}

Figure 5(a) presents a sample of eight elementary units that provide a phase shift over a $2 \pi$ span with a step of $\pi / 4$. The units are fabricated with thermoplastics via 3D printing (Stratasys Dimension Elite, $0.08 \mathrm{~mm}$ in precision).

We experimentally illustrate the performance of our units by implementing them to form a self-bending beam illustrated in Fig. 1. The self-bending beam is in the nonparaxial regime that can challenge the performance of our passive array. The beam was recently formed by using an active phased array [26,27], where the complexity

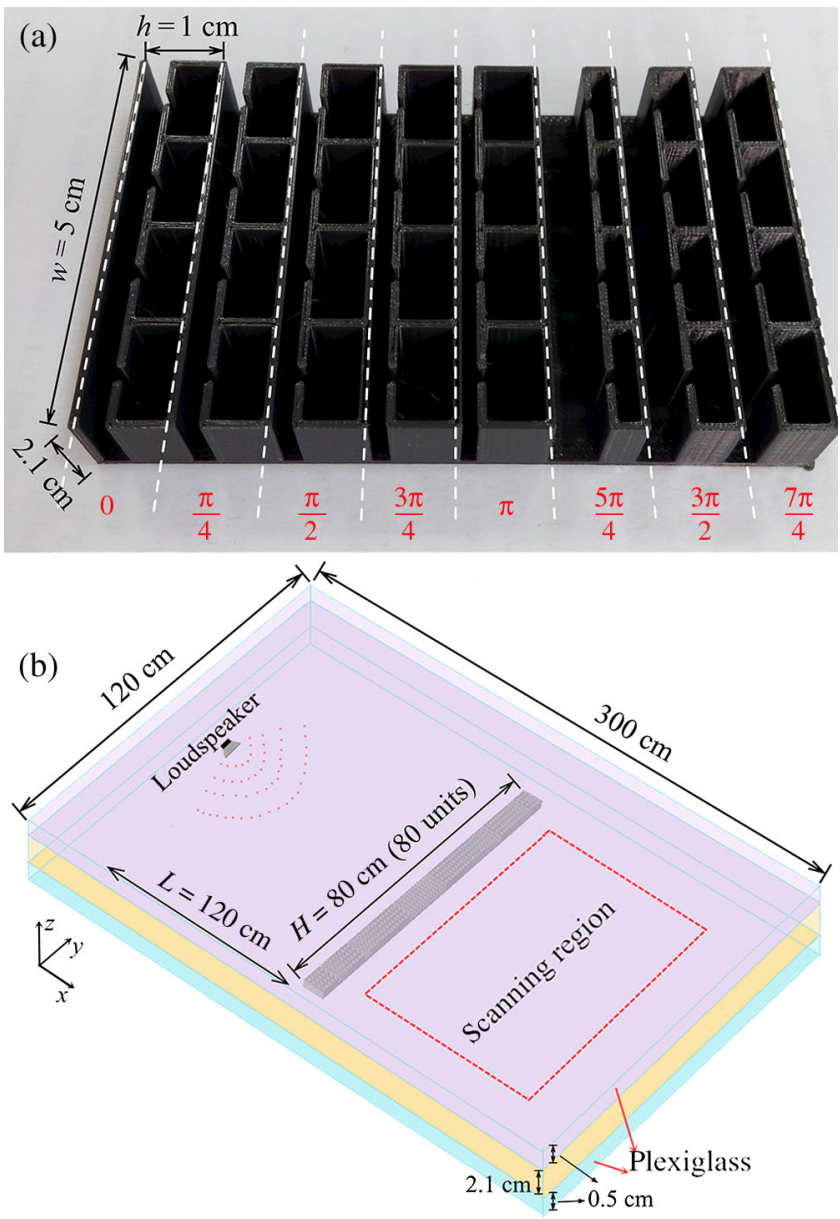

FIG. 5. Experimental samples and setup. (a) Fabricated samples of the eight elementary units for varying the phase from 0 to $2 \pi$ with a step of $\pi / 4$; the sample's dimension is for sound waves of resonant wavelength $\lambda=10 \mathrm{~cm}$ (viz., frequency $f_{0}=3430 \mathrm{~Hz}$ in air). (b) Experimental setup for shaping monochromatic sound of frequency $f_{0}$ from a loudspeaker to form a wave front as in Fig. 1 by using an 80 -unit screen sandwiched between two plexiglass plates $\left(300 \times 120 \times 0.5 \mathrm{~cm}^{3}\right.$ of a $2.1-\mathrm{cm}$ gap. Wedgeshaped sound-absorbing foam is installed at the boundaries of the plates to mimic an anechoic environment. The speaker dimension is $3 \times 3 \mathrm{~cm}^{2}$, and sound fields are measured in the scanning region using a 1/4-in. microphone (Brüel \& Kjær type 4961); both magnitude and phase are recorded by referring to a signal recorded by a second microphone at a fixed point. 

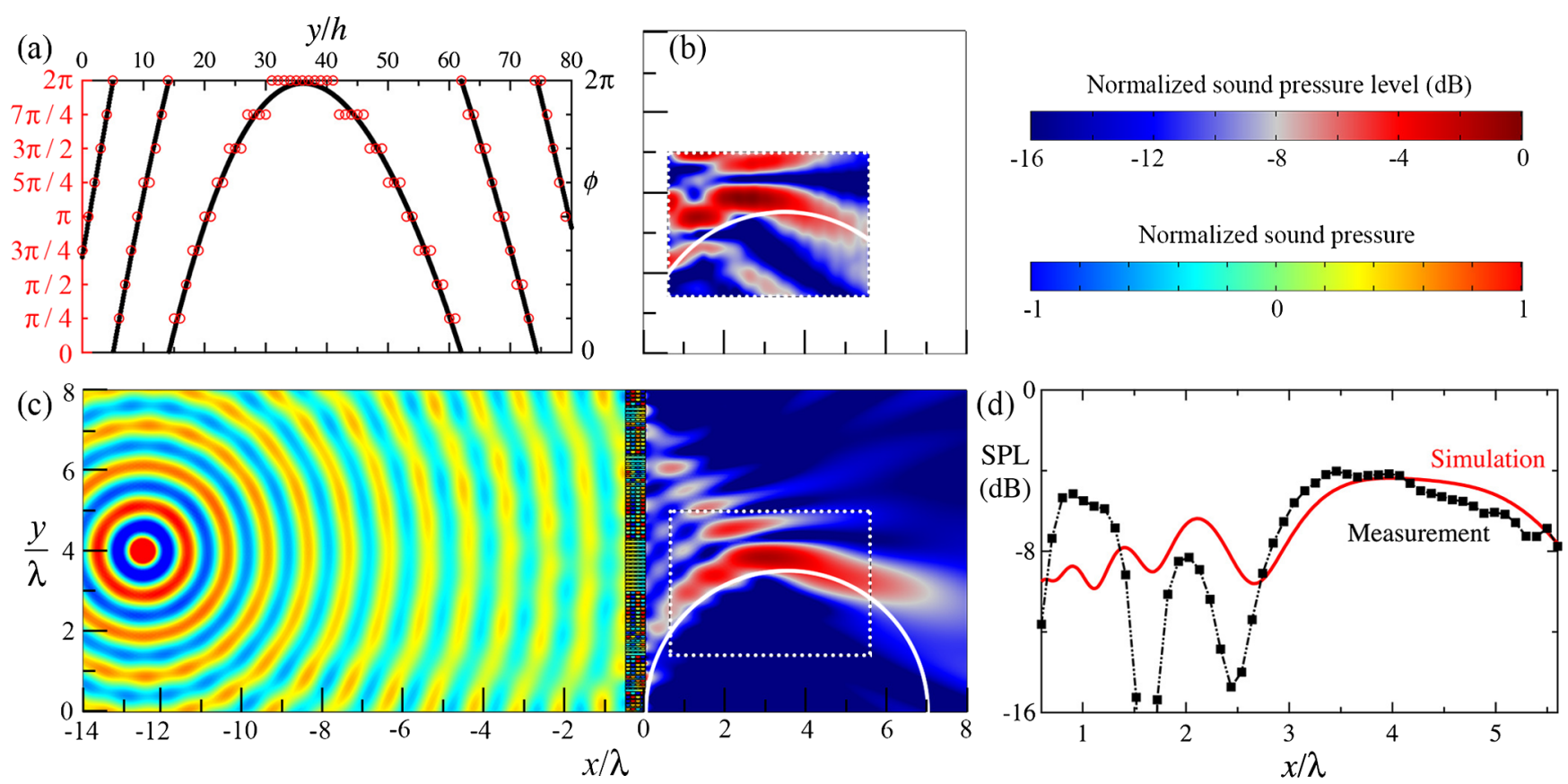

FIG. 6. Experimental realization of a nonparaxial self-bending beam using a screen of 80 units. (a) Distribution of the 80 elements along the screen as indicated by their discrete phase shift (the 80 red hollow circles) resembling the desired phase profile $\phi(y)$ (black curves; see text for detail). (b) Self-bending beam measured in the scanning region of Fig. 5(b), where the white circle denotes the desired sound path. (c) Sound field simulated for the 80 elements and loudspeaker, where the field at $x>0$ is sound pressure level [(SPL) normalized by the maximum value] and at $x<0$ is a snapshot of normalized sound pressure. The white dotted rectangular region refers to the area where experimental measurements are taken. (d) Comparison of the experimental measurements with numerical simulation for the beam along the white-colored trajectory in (b) and (c).

was the control of the individual sources of the active array. Here we can simply use a passive metascreen to form this nonparaxial beam. Our screen has a length of $8 \lambda$ [cf. Fig. 5(b)], composed of 80 elementary units (recall that each unit occupies $\lambda / 10$ along the screen) for the purpose of forming a reasonably good wave front.

The distribution of the 80 units along the screen is illustrated by the discrete phase shift shown by red hollow points in Fig. 6(a). The discrete phase shift is determined from the desired continuous phase profile [cf. black curves in Fig. 6(a)],

$$
\phi(y)=-k(y-2 r \sqrt{y / r})-k\left[\sqrt{(y-H / 2)^{2}+L^{2}}-L\right],
$$

which is the profile in Fig. 1 but with an additional term accounting for the phase of the source (located at $L=120 \mathrm{~cm}$ away from the screen) while arriving at the screen of length $H=80 \mathrm{~cm}$ [cf. Fig. 5].

The screen shapes the sound field of the point source into a self-bending beam that is measured and shown in Fig. 6(b). The measured field reveals the structures' ability to form such a self-bending beam beyond the paraxial approximation. For the purpose of comparison, Fig. 6(c) shows sound fields simulated for the 80-unit screen and loudspeaker [28]. The comparison of the simulation with experimental measurements is further illustrated in Fig. 6(d) showing the sound pressure level along the designed arc trajectory, where the difference in the near field stems from imperfect sound absorption by wedgeshaped foams at two edges of the metascreen that leads to standing waves in between. We, hence, demonstrate the performance of our metascreen even in forming such a beam in the nonparaxial approximation.

\section{SUMMARY AND OUTLOOK}

To summarize, we design and realize a metascreen-based passive array that efficiently transmits sound energy from a primary source, steers a local phase shift that spans a full $2 \pi$ range, and, meanwhile, has a deep subwavelength spatial resolution. The elements of the array have a hybrid structure composed of a series of Helmholtz resonators and Fabry-Perot resonant straight pipes. A series connection of Helmholtz resonators possess the ability to yield a $2 \pi$ range phase shift while the Fabry-Perot resonance maintains the impedance matching to enhance the sound transmission. The element's discrete resolution is as small as $1 / 10$ of the sound wavelength, allowing a sufficiently fine control of the sound fields beyond the paraxial region, as we demonstrate in experiment for generating a nonparaxial self-bending beam. 
Constructing a deep subwavelength structure for high transmission and a full range of phase shift is a challenge in acoustic wave-front steering. By resolving this challenge, our designed metascreen has the capability to play a role like conventional phased arrays but in a passive way that avoids the complexity of active arrays. Our passive array using a metascreen with its simple configuration and extreme acoustic performance can, hence, have applications in places where the conventional array will have complexity and limited capability. The screen may be used for sound-field shaping in underwater sound arrays [29], nondestructive testing [30], audio presentation [31], and particle manipulation [32]. The screen may also be used in ultrasound imaging and therapy to create a beam without diffraction [33] or focused at a specific depth below the acoustic transducer [34].

Our passive phased array provides a finite-band control of a transmitted acoustic wave front. In the future, a complementary design for the steering of broadband pulses will be valuable.

\section{ACKNOWLEDGMENTS}

We thank Dr. Xin-ye Zou at the Nanjing University for discussions. Y. L. acknowledges the support of Professor Ping Sheng at Hong Kong University of Science and Technology and Professor Badreddine Assouar at Université de Lorraine. L. Z. acknowledges the support of the 2013-14 F. V. Hunt Postdoctoral Research Fellowship of the Acoustical Society of America. Financial support by the National Basic Research Program of China (973 Program, Grants No. 2010CB327803 and No. 2012CB921504), the National Natural Science Foundation of China (Grants No. 11174138, No. 11174139, No. 11222442, No. 81127901, and No. 11274168), the Program for New Century Excellent Talents in University (Grant No. NCET12-0254), and the Priority Academic Program Development of Jiangsu Higher Education Institutions are gratefully acknowledged.

Y. L. and X. J. contributed equally to this work.

[1] J. Durnin, J. J. Miceli, and J. H. Eberly, Diffraction-Free Beams, Phys. Rev. Lett. 58, 1499 (1987).

[2] G. A. Siviloglou, J. Broky, A. Dogariu, and D. N. Christodoulides, Observation of Accelerating Airy Beams, Phys. Rev. Lett. 99, 213901 (2007).

[3] J. F. Nye and M. V. Berry, Dislocations in wave trains, Proc. R. Soc. A 336, 165 (1974).

[4] D. H. Johnson and D. E. Dudgeon, Array Signal Processing: Concepts and Techniques (Prentice-Hall, Englewood Cliffs, NJ, 1993).

[5] A. M. Weiner, Femtosecond pulse shaping using spatial light modulators, Rev. Sci. Instrum. 71, 1929 (2000).
[6] N. Yu, P. Genevet, M. A. Kats, F. Aieta, J. P. Tetienne, F. Capasso, and Z. Gaburro, Light propagation with phase discontinuities: Generalized laws of reflection and refraction, Science 334, 333 (2011).

[7] S. Sun, Q. He, S. Xiao, Q. Xu, X. Li, and L. Zhou, Gradientindex meta-surfaces as a bridge linking propagating waves and surface waves, Nat. Mater. 11, 426 (2012).

[8] F. Monticone, N. M. Estakhri, and A. Alù, Full Control of Nanoscale Optical Transmission with a Composite Metascreen, Phys. Rev. Lett. 110, 203903 (2013).

[9] P. M. Morse and K. U. Ingard, Theoretical Acoustics (Princeton University Press, Princeton, NJ, 1986).

[10] Z. Liu, X. Zhang, Y. Mao, Y. Y. Zhu, Z. Yang, C. T. Chan, and P. Sheng, Locally resonant sonic materials, Science 289, 1734 (2000).

[11] N. Fang, D. Xi, J. Xu, M. Ambati, W. Srituravanich, C. Sun, and $X$. Zhang, Ultrasonic metamaterials with negative modulus, Nat. Mater. 5, 452 (2006).

[12] Z. Yang, J. Mei, M. Yang, N. H. Chan, and P. Sheng, Membrane-Type Acoustic Metamaterial with Negative Dynamic Mass, Phys. Rev. Lett. 101, 204301 (2008).

[13] J. Christensen and F. J. García de Abajo, Anisotropic Metamaterials for Full Control of Acoustic Waves, Phys. Rev. Lett. 108, 124301 (2012).

[14] Z. Liang and J. Li, Extreme Acoustic Metamaterial by Coiling Up Space, Phys. Rev. Lett. 108, 114301 (2012).

[15] C. Shen, J. Xu, N. X. Fang, and Y. Jing, Anisotropic Complementary Acoustic Metamaterial for Canceling Out Aberrating Layers, Phys. Rev. X 4, 041033 (2014).

[16] Y. Li, B. Liang, Z.-m. Gu, X.-y. Zou, and J.-c. Cheng, Reflected wavefront manipulation based on ultrathin planar acoustic metasurfaces, Sci. Rep. 3, 2546 (2013).

[17] J. Zhao, B. Li, Z. Chen, and C.-W. Qiu, Manipulating acoustic wavefront by inhomogeneous impedance and steerable extraordinary reflection, Sci. Rep. 3, 2537 (2013).

[18] Y. Li, X. Jiang, R. Q. Li, B. Liang, X. Y. Zou, L. L. Yin, and J. C. Cheng, Experimental Realization of Full Control of Reflected Waves with Subwavelength Acoustic Metasurfaces, Phys. Rev. Applied 2, 064002 (2014).

[19] G. Ma, M. Yang, S. Xiao, Z. Yang, and P. Sheng, Acoustic metasurface with hybrid resonances, Nat. Mater. 13, 873 (2014).

[20] Y. Xie, W. Wang, H. Chen, A. Konneker, B. I. Popa, and S. A. Cummer, Wavefront modulation and subwavelength diffractive acoustics with an acoustic metasurface, Nat. Commun. 5, 5553 (2014).

[21] K. Tang, C. Qiu, M. Ke, J. Lu, Y. Ye, and Z. Liu, Anomalous refraction of airborne sound through ultrathin metasurfaces, Sci. Rep. 4, 6517 (2014).

[22] J. Mei and Y. Wu, Controllable transmission and total reflection through an impedance-matched acoustic metasurface, New J. Phys. 16, 123007 (2014).

[23] L. Rayleigh, The Theory of Sound, 2nd ed. (Dover, New York, 1945).

[24] M. Molerón, M. Serra-Garcia, and C. Daraio, Acoustic fresnel lenses with extraordinary transmission, Appl. Phys. Lett. 105, 114109 (2014). 
[25] Simulations are conducted with COMSOL multiphysics software. The simulated materials for HRs have a density $1180 \mathrm{~kg} / \mathrm{m}^{3}$ and sound speed $2700 \mathrm{~m} / \mathrm{s}$, which are the parameters of the $3 \mathrm{D}$ printed materials in the experiments. The surrounding medium is air with density $1.21 \mathrm{~kg} / \mathrm{m}^{3}$ and sound speed $343 \mathrm{~m} / \mathrm{s}$.

[26] P. Zhang, T. Li, J. Zhu, X. Zhu, S. Yang, Y. Wang, X. Yin, and $X$. Zhang, Generation of acoustic self-bending and bottle beams by phase engineering, Nat. Commun. 5, 4316 (2014).

[27] S. Zhao, Y. Hu, J. Lu, X. Qiu, J. Cheng, and I. Burnett, Delivering sound energy along an arbitrary convex trajectory, Sci. Rep. 4, 6628 (2014).

[28] We ignore the viscous effect in our simulations because the thickness of the boundary layer is only $4 \times 10^{-4}$ of the wavelength for the simulated sound fields of $3430 \mathrm{~Hz}$.
[29] C. H. Sherman and J. L. Butler, Transducers and Arrays for Underwater Sound (Springer, New York, 2007).

[30] B. W. Drinkwater and P. D. Wilcox, Ultrasonic arrays for non-destructive evaluation: A review, NDT\&E Int. 39, 525 (2006).

[31] J. Ahrens, R. Rabenstein, and S. Spors, Sound field synthesis for audio presentation, Acoust. Today 10, 15 (2014).

[32] F. Zheng, Y. Li, H. S. Hsu, C. G. Liu, C. T. Chiu, C. Lee, H. H. Kim, and K. K. Shung, Acoustic trapping with a high frequency linear phased array, Appl. Phys. Lett. 101, 214104 (2012).

[33] J.-Y. Lu and J.F. Greenleaf, Ultrasonic nondiffracting transducer for medical imaging, IEEE Trans. Ultrason. Ferroelectr. Freq. Cont. 37, 438 (1990).

[34] D. Pajek and K. Hynynen, Applications of transcranial focused ultrasound surgery, Acoust. Today 8, 8 (2012). 